\title{
A fresh pair of eyes on prospective memory monitoring
}

\author{
Jill Talley Shelton $^{1,2} \cdot$ Eddie A. Christopher $^{1,2}$
}

Published online: 11 March 2016

(C) Psychonomic Society, Inc. 2016

\begin{abstract}
Remembering to complete one's future intentions is termed prospective memory. We employed a new eyetracking paradigm to concretely observe the impact of environmental cues on strategic monitoring within a visual prospective memory task. Participants worked on a continuous living-count task comprising images, while simultaneously being asked to respond to a prospective memory target when it appeared. Importantly, the prospective memory target appeared in a different area of the participant's visual field than did the continuous task, which is consistent with prospective memory in many real-world situations, and further allows for a clear index of strategic monitoring processes. Subtle cues in the form of semantically related images were embedded in the continuous task to prompt monitoring for the prospective memory target. Overt strategic monitoring was operationalized as the number of times participants fixated on the designated target area, and cue-driven monitoring was defined by the number of fixations on the prospective memory target region directly after fixating on a related cue. Overt strategic monitoring for the prospective memory target was directly observed for participants in the prospective memory condition, and cue-driven monitoring was also observed in these participants, since they were more likely to initiate monitoring immediately after fixating on a semantically related cue, relative to an unrelated cue. This psychophysiological approach afforded precise measurement of the strategic monitoring process and revealed
\end{abstract}

Jill Talley Shelton

jill-shelton@utc.edu

1 Department of Psychology, Lee University, Cleveland, TN, USA

2 Department of Psychology, University of Tennessee, 615 McCallie Ave, Chattanooga, TN 37403, USA how contextual cues in the environment interact with the cognitive mechanisms supporting prospective memory.

Keywords Prospective memory - Overt strategic monitoring · Cue-driven monitoring $\cdot$ Context $\cdot$ Preparatory attention

An important aspect of daily life is remembering to execute future intentions at the appropriate time or in response to a designated event, which is termed prospective memory. Realworld prospective memory tasks include activities such as remembering to turn off the oven or to take blood pressure medication. Prospective memory failures can produce devastating consequences in some real-world environments, such as pilot and air traffic control navigation (Dismukes, 2008). Laboratory studies have suggested that the observed impairments in prospective memory, such as those seen in air traffic control simulations (Loft \& Remington, 2010) or in Parkinson's disease patients (Foster, McDaniel, Repovš, \& Hershey, 2009), might stem from deficient use of the strategic monitoring process that often supports retrieval of a future intention. The strategic monitoring process is characterized by shifting attention from current ongoing activities in order to search for the appropriate time to execute the future intention (McDaniel \& Einstein, 2000). Furthermore, the monitoring process has been characterized as a preparatory attention process that is critical for the successful retrieval of prospective memory intentions (Smith, 2003). The methods used for assessing strategic monitoring processes in the laboratory are, however, somewhat imprecise and have recently come under scrutiny (Horn, Bayen, \& Smith, 2011; Loft, Humphreys, \& Whitney, 2008; McDaniel, LaMontagne, Beck, Scullin, \& Braver, 2013; Smith, 2010). In the present study, we used eyetracking technology to more directly observe the overt strategic monitoring that supports prospective memory and 
to flesh out the degree to which environmental context will trigger prospective memory monitoring.

In the typical laboratory event-based prospective memory paradigm, participants are engaged in a continuous ongoing activity (e.g., making word/nonword judgments for strings of letters presented on a computer screen), while they are simultaneously given the prospective memory demand to make a designated response when an infrequently presented target item appears during the ongoing task (Einstein et al., 2005). The stimuli used for the ongoing task and the prospective memory task are typically one and the same - a situation akin to needing to remember to change the channel at the end of the television show one is currently watching. On the other hand, many real-world prospective memory demands require an individual to shift attention to a different stimulus than the one relevant to their ongoing task. For example, an individual watching a news segment but wishing to monitor the ticker for a particular news update must momentarily cease watching the segment and direct attention to the bottom of the screen to perform their prospective memory task. In the present study we will explore prospective memory in this second context, wherein the ongoing task and the prospective memory target are distinctly different stimuli. This approach will not only enable an evaluation of a specific kind of event-based prospective memory task that is not typically examined in the laboratory, but will also enable a clear assessment of how overt strategic monitoring processes are used in support of prospective remembering. We use the term overt to note our focus on the reallocation of attention (either conscious or unconscious) from the ongoing task to the prospective memory demand.

Strategic monitoring is typically measured by the amount of slowing observed on the continuous ongoing activity when a prospective memory task is embedded, relative to when no prospective memory demand is present (referred to in the literature as the ongoing-task cost or task interference). Studies have demonstrated that the presence of a prospective memory demand (relative to when no demand is present) can lead to an ongoing-task cost (Einstein et al., 2005; Marsh, Hicks, Cook, Hansen, \& Pallos, 2003; Park, Hertzog, Kidder, Morrell, \& Mayhorn, 1997; Smith, 2003), and the observed cost is believed to reflect the strategic monitoring process. Identifying the processes that support prospective memory has important theoretical implications; however, there is rising concern regarding what cognitive processes are being tapped by measures of the ongoing-task cost (Hicks, Marsh, \& Cook, 2005; Horn, Bayen, \& Smith, 2011; Loft et al., 2008; Smith, 2010). For example, Meier and Rey-Mermet (2012) demonstrated that the ongoing-task cost is sometimes reflective of an aftereffect of completing a prospective intention, not of interference from preparatory monitoring processes. Similar criticisms have arisen from empirical work that has suggested that the ongoing task cost can be ameliorated if ample response time is afforded to participants when a prospective memory target is encountered (Loft \& Remington, 2013). It is likely that the ongoing-task cost reflects factors other than individuals strategically monitoring for a prospective memory cue. Recent neuroimaging evidence has supported this claim, by demonstrating that the typical measure of ongoing task cost can occur without any evidence of sustained brain activity (e.g., in the anterior prefrontal cortex) that would be expected if individuals were engaging an attentionally demanding monitoring process (McDaniel et al., 2013). Alternative accounts of task interference have posited that observed ongoing-task costs may reflect metacognitive processes that lead individuals to adjust how they allocate attention between the ongoing and prospective memory tasks (Hicks et al., 2005; Rummel \& Meiser, 2013). Rummel and Meiser recently demonstrated that increases in the ongoing-task cost reflect metacognitive expectations regarding the difficulty of the prospective memory task. Furthermore, metacognitive expectations moderated whether task interference was even functionally related to prospective memory performance. For strategic monitoring to be considered functionally related to performance, the expectation is that an ongoing-task cost should be associated with better prospective memory performance (Scullin, McDaniel, \& Einstein, 2010); however, a growing number of studies have failed to observe such a relationship across prospective memory tasks (Einstein et al., 2005; Harrison \& Einstein, 2010; McNerney \& West, 2007; Scullin, McDaniel, \& Einstein, 2010; Scullin, McDaniel, \& Shelton, 2013; Wang et al., 2011). These findings may reflect the presence of poorer-quality strategic monitoring processes or the presence of spontaneous retrieval processes; however, they may also be indicative of potential limitations in the typical measure of strategic monitoring.

In this study, we developed a prospective memory task using an eyetracker that would allow for isolating the overt strategic monitoring process that is theorized to support prospective remembering. Specifically, we were able to concretely observe the overt strategic monitoring process by tracking participants' eye fixations to a region of the visual field that was designated for upcoming prospective memory targets. Prior research suggested that this kind of approach is useful for capturing overt strategic monitoring processes, even when they may be missed by traditional measures of monitoring. Brown, Hicks, and Beck (2014) found that participants with a prospective memory intention fixated on the prospective memory target area significantly more often than did control (no prospective memory) participants, and that the effect was stronger when increasing emphasis was placed on the prospective memory task relative to the ongoing task. Notably, participants with the prospective memory intention did not show significant slowing to the ongoing task relative to their control counterparts. We used a similar approach in the present study to achieve our primary goal of examining whether 
attention to contextual factors in the environment can prompt retrieval of the future intention, leading to cue-driven monitoring.

Both self-report and behavioral data suggest that environmental cues can serve as a reminder for a prospective memory intention (Harris \& Wilkins, 1982; Henry, Rendell, Phillips, Dunlop, \& Kliegel, 2012; Kvavilashvili \& Fisher, 2007) and that environmental cues can be used to support prospective memory in laboratory simulated air traffic control scenarios (Loft, Smith, \& Bhaskara, 2011). It remains unclear exactly how environmental cues interact with the cognitive mechanisms supporting prospective memory, although some research has suggested that people will adjust how they allocate attention to the prospective memory task if they are instructed about contextual cues that will accompany the prospective memory target (Kuhlmann \& Rummel, 2014). Scullin, McDaniel, and Einstein (2010) examined this question by implanting subtle cues (either words that were semantically related to the prospective memory target or specific color backgrounds) proximal to the onset of that target item. In this study, participants were engaged in a typical laboratory task in which the ongoing task required word-nonword decisions to be made, and the prospective memory task was to make a designated response every time a particular item appeared. Ongoing-task costs were examined to determine whether the environmental cue would prompt monitoring and whether the cost was functionally related to prospective memory performance. Interestingly, environmental cues were only effective in prompting monitoring in certain prospective memory task situations, and the cost was only functionally related to prospective memory performance in one of four experimental conditions (cued, nonfocal condition). The findings from Scullin and colleagues are important to the goals of the present research for two reasons: (1) They raised questions about the utility of subtle contextual cues and the probability that these cues would prompt strategic monitoring for prospective memory targets, and (2) they highlighted the potential limitations of traditional measures of strategic monitoring, since this index was not functionally related to prospective memory performance in most conditions.

The approach used in the present study extends our understanding of how contextual cues impact the processes supporting prospective memory by isolating the temporal onset of overt strategic monitoring. To this end, participants worked on a visual search task in which they counted the number of living objects that appeared in a visual array. Half of the participants were also given a prospective memory demand that required them to make a designated response (i.e., to say "hit") when they saw a specific target object (an apple image). The prospective memory target appeared infrequently and in a different area of the participant's visual field than the continuous task. Thus, the index of strategic monitoring was the total number of times participants in the prospective condition switched their visual gaze from the ongoing activity to fixate on the prospective-memory-designated area of the visual field, relative to control participants who were not told about the prospective memory task. Images were also embedded in the ongoing task that were semantically related to the prospective memory target, to determine whether fixation on these cues would prompt cue-driven monitoring for participants in the prospective memory condition. We predicted that the new index of monitoring we developed would enable the direct observation of cue-driven monitoring.

\section{Method}

\section{Participants}

The participants $(n=52)$ were undergraduate students at Lee University (75\% female, $84.6 \%$ Caucasian; $M_{\text {age }}=20.5$ years). All participants had either normal or corrected-tonormal vision and were awarded extra credit in a course of their choosing. A $2 \times 2$ mixed-factor design was employed. Participants were randomly assigned to either the experimental condition $(n=26)$, which received a prospective memory demand, or a control condition $(n=26)$ that was never given the prospective memory intention. The within-participants manipulation was the presence of cues during the ongoing task that were either semantically related to the prospective memory target or unrelated to the target.

\section{Apparatus}

A Tobii T120 eyetracker was used for data collection. It is typically accurate within $0.5 \mathrm{deg}$ and has a resolution of 0.3 deg. All visual angle metrics are reported in degrees. Visual scan patterns were recorded at a rate of $120 \mathrm{~Hz}$. The Tobii had an 18-in. monitor with a screen resolution of $1,024 \times 768$ pixels; the prospective memory target area represented $1.6 \%$ of this screen, giving it a visual angle of $0.688^{\circ}$. However, semantically related areas of interest took up $6.5 \%$ of the screen (visual angle $=2.793^{\circ}$ ), with unrelated areas of interest accounting for $7.2 \%$ (visual angle $=3.093^{\circ}$ ) of the screen. Participants were seated approximately 24 in. from the Tobii monitor. The precise distance was allowed to vary slightly between participants, as a chin rest was not used. Before testing, the eyetracker was calibrated to measure each participant's eye movements.

\section{Materials}

The ongoing task was a visual search in which participants counted the number of living objects on the screen for each trial. The living-count trials consisted of an array of images, half of which were living (e.g., dog) and the other half of 


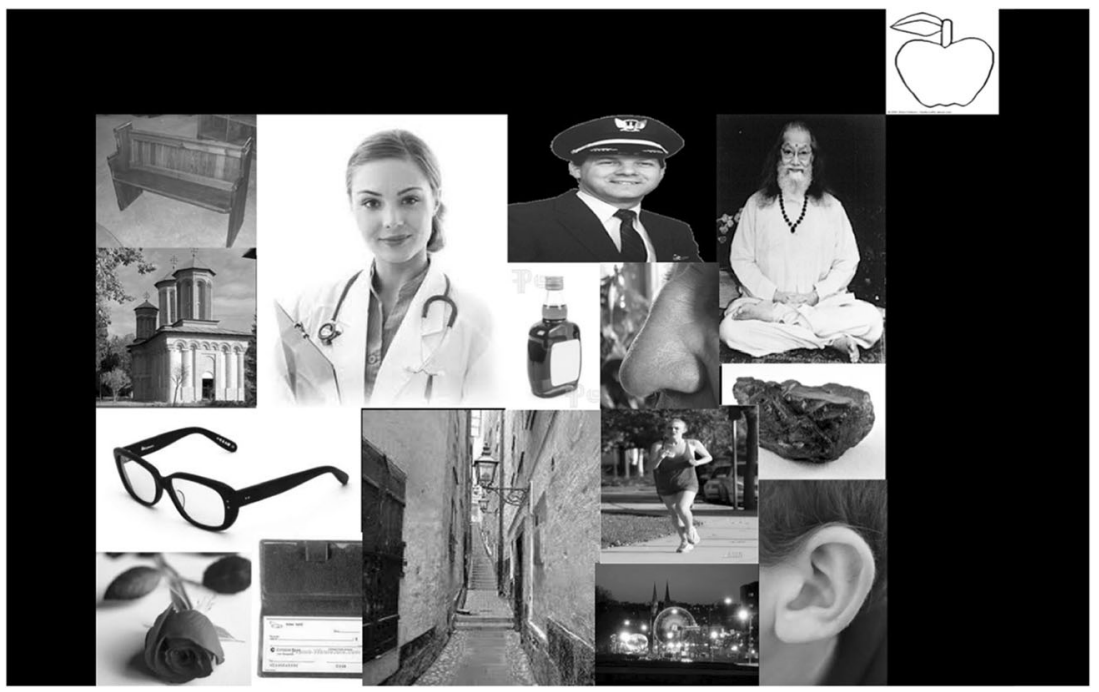

Fig. 1 Example of a subtrial

which were nonliving (e.g., chair). Each image array formed a rectangle and contained approximately 20 small black-andwhite images. In addition, another set of images were obtained to form the prospective memory target region. The target region was a rectangle, distinctly removed from the living-count task area, in which one image appeared at a time. This separate region of the screen was the location where the prospective memory targets appeared (see Fig. 1 for an example slide). Importantly, the images presented in the prospective memory target region changed three times as often as the images in the living-count task. Each trial of the living-count task was timed at $9 \mathrm{~s}$, whereas the image in the prospective memory target region changed every $3 \mathrm{~s}$ (referred to as subtrials). The purpose of having the images in the prospective memory region change was to encourage overt strategic monitoring for targets in the experimental condition, which is often required in realworld visual scenes, which are more dynamic in nature. Thus, there were three subtrials per trial over a total of 40 livingcount trials (120 subtrials). Imposing a time limit for each trial was critical in the present study, as this encouraged participants to be strategic about balancing the demands of the living-count and prospective memory tasks (for those in the experimental condition). The timing of trials was determined through pilot testing, so that most people could finish (or come close to finishing) the living count for each slide, although performance on the living-count task was not of key interest in the present study.

In addition, some images presented in the living-count task were either semantically related (e.g., a banana) or unrelated (e.g., a duck) to the prospective memory target (an apple). These prospective memory cues were present in ten of the 40 trials, whereas unrelated cues were present in a separate ten of the 40 trials. We examined the Nelson, McEvoy, and Schreiber (1998) free association norms to determine what words were associated with apple. Images that matched these words were present in ten of the task trials. The related images consisted of assorted fruits and berries, trees, and worms. Although the same types of cues were used repeatedly, the exact images were never repeated. The method that we used to designate the unrelated cues was to identify images that were not semantically related to the word apple and that were in a location similar to that of the related cue on trials proximal to related-cue trials.

\section{Procedure}

After providing informed consent, participants were seated at the eyetracking computer and told to remain still and keep their eyes on the screen. They were instructed to count the number of living objects they could find on each trial and to verbally report this number to the experimenter. They were further informed that there was a time limit on each trial. Participants in the experimental condition were told that in addition to performing the living-count task, they needed to be mindful of the box in the top right-hand corner of the screen, which would contain images changing at three times the speed of the trial. The transition between subtrials occurred every $3 \mathrm{~s}$ and was made as seamless as possible, to reduce the likelihood of the change being noticed unless participants were fixating on the box in the top right-hand corner. ${ }^{1}$ The prospective memory target was present in four randomly

\footnotetext{
${ }^{1}$ We calculated the mean number of times that participants fixated on regions other than the prospective memory target region immediately following the start of a new subtrial, to determine whether the change of subtrials attracted attention to the target region. Across the 120 subtrials, participants fixated on an average of 3.78 regions other than the prospective memory target region before fixating on that target region, which is significantly different from zero, $t=74.81, p<.001$. These data are consistent with the claim that the subtrial change was likely to be unnoticed by participants.
} 
selected subtrials out of the 120 , and never twice in the same trial (the target appeared at least once in each of the three subtrial positions of a given target trial). After being read the instructions, participants were asked to repeat the instructions back to the researcher to demonstrate that they had been properly encoded. At this point, participants were allowed to practice the living-count task.

Participants then completed a vocabulary test as their filler task before continuing with the experiment. The vocabulary test took them approximately $3 \mathrm{~min}$ to complete. Participants then began the living-count task with no further reminder of the prospective memory task. The living-count task took approximately $6 \mathrm{~min}$ to complete. Next, participants were instructed to perform a letter-number sequencing task, which measures working memory capacity (Gold, Carpenter, Randolph, Goldberg, \& Weinberger, 1997). The purpose of including the vocabulary test was simply to serve as a delay between the encoding of the prospective memory instructions and the performance interval. The letter-number sequencing task was included to assess whether overt monitoring or prospective memory performance was related to individual differences in working memory capacity. These individual difference measures did not correlate with prospective memory or with strategic monitoring, and therefore will not be discussed further.

\section{Results}

\section{Monitoring}

We characterized overt monitoring in two ways: as total monitoring and cue-driven monitoring. Total monitoring was operationalized as the total number of times participants fixated on the prospective memory target region across all of the task trials. Cue-driven monitoring was operationalized as the total number of times participants fixated on specified cues (related and unrelated) during the living-count task and immediately shifted their gaze from those cues to the prospective memory target region. Thus, both dependent measures represent frequency counts of the total number of fixations on the designated target region, but only the cue-driven monitoring index is concerned with what images were fixated prior to fixating on the prospective memory target region.

Total monitoring We employed an independent-samples $t$ test to assess the total monitoring activity between conditions (experimental vs. control). Indeed, a significant difference between conditions was observed, $t(50)=10.43, p<.001$. This effect reflected that participants in the experimental condition $(M=87.85, S D=36.01)$ fixated on the prospective memory target region eight times more often than those in the control condition $(M=11.00, S D=10.66)$.
Cue-driven monitoring Next we sought to understand the extent to which monitoring was affected by the cues. A 2 (Condition: experimental/control) $\times 2$ (Cue: related/unrelated $)$ mixed-model analysis of variance (ANOVA) was used to explore the effect of cues on monitoring between conditions. A significant effect of cue type was observed, $F(1,50)=18.86$, $M S E=2.15, p<.001, \eta^{2}=.22$, and this effect arose from related cues $(M=2.02, S D=2.67)$ eliciting more monitoring than unrelated cues $(M=0.77, S D=1.06)$ overall. The main effect of cue was qualified by a significant Condition $\times$ Cue interaction, $F(1,50)=17.72, M S E=2.15, p<.001, \eta^{2}=.20$. This interaction was expected, and we followed it up with additional tests for simple effects.

First, an independent-samples $t$ test was used to directly compare the levels of monitoring following a related cue between the conditions (experimental vs. control). The two conditions did differ significantly, $t(50)=6.25, p<.001$ : Participants in the experimental condition were much more likely to engage in monitoring after fixating on a related cue than were control participants. Participants within the experimental condition also monitored after a related cue significantly more often than they monitored following an unrelated cue, $t(25)=4.37, p<.001$. Furthermore, there was no difference in the monitoring frequencies following related and unrelated cues within the control condition, $t(25)=0.33, p=.75$. This pattern of results supported our hypothesis that related cues would trigger monitoring activity significantly more often than unrelated cues, though only for those with the prospective memory intention (see Fig. 2). Indeed, the participants in the control condition demonstrated virtually no evidence of cue-driven monitoring.

\section{Prospective memory performance}

Prospective memory performance was operationalized as the proportion of times participants said "hit" within one trial of an apple picture appearing. Prospective memory performance was moderate $(M=.60, S D=.35)$ and was found to be positively correlated to total monitoring activity $(r=.47, p=.01)$.

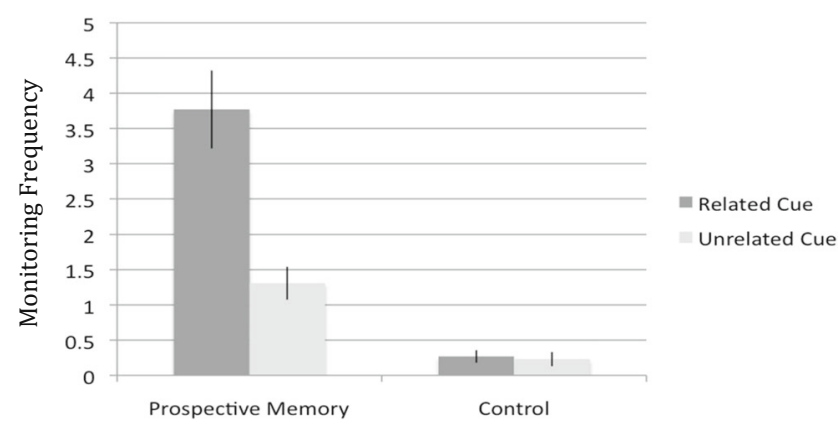

Fig. 2 Frequency of cue-driven monitoring by condition. Error bars represent one standard error of the mean 


\section{Ongoing-task performance}

Participants were told to verbally report the number of living objects they saw in each trial; thus, performance was operationalized as the number of trials on which the correct number of living objects was reported. An independent-samples $t$ test revealed a marginally significant difference between the experimental $(M=5.00, S D=3.99)$ and control $(M=7.69$, $S D=5.98)$ conditions, $t(50)=1.91, p=.06$. The group difference was in the anticipated direction, with the experimental group performing worse than the control group.

\section{Discussion}

The goal of this study was to develop a more direct measure of the strategic monitoring process theorized to support prospective memory retrieval, in order to evaluate the impact of contextual cues on the cognitive mechanisms supporting prospective memory. A notable strength of the task developed for the present study is that, unlike the traditional laboratory-based prospective memory task, it captures the common occurrence in real-world settings of having to overtly shift attention to other areas of the visual scene to monitor for upcoming target events. This, in turn, enabled a more precise estimate of overt strategic monitoring than is typically afforded by behavioral measures. The sheer magnitude of the observed effects in the present study provides unequivocal evidence of the strategic monitoring or preparatory attention processes that have been theorized to support retrieval in prospective memory tasks (McDaniel \& Einstein, 2000; Smith, 2003). Specifically, we found that participants who were given the prospective memory intention were more likely to shift their visual attention from the ongoing task to the area of the screen where the prospective memory target occasionally appeared. Of particular interest was the finding that a cue-driven monitoring process was directly initiated by subtle cues, in the form of semantically related images that were embedded in the continuous task. Past studies have revealed that contextual cues in the environment often prompt retrieval of a future intention (Harris \& Wilkins, 1982, Henry et al., 2012; Kuhlmann \& Rummel, 2014; Kvavilashvilli \& Fisher, 2007), and the present research extended this finding with the observation that contextual cues can trigger retrieval of the future intention, thereby prompting participants to overtly monitor for prospective memory targets.

Salient external cues have been shown to be helpful to adults (Guajardo \& Best, 2000) in a variety of laboratory studies (Einstein \& McDaniel, 1996; Kim \& Mayhorn, 2008; Villa, 1998), including investigations of air traffic control (Loft et al., 2011). In particular, external aids were helpful when they appeared in the context of the prospective memory targets. These external aids led to a reduction in the ongoing-task cost, in addition to boosting prospective memory performance. Of particular interest to the present study is the impact of more subtle, implicit cues on the cognitive processes theorized to support prospective memory.

The newly developed dynamic multiprocess framework (Scullin et al., 2013) postulates that contextual cues can prompt spontaneous retrieval of the future intention and lead individuals to begin strategically monitoring for the upcoming prospective memory target. Past studies have provided partial support for these claims by demonstrating that monitoring behavior increased (at least in some experimental conditions) when subtle cues were embedded in the ongoing task (Scullin et al., 2010) and that activity in brain regions associated with strategic monitoring (i.e., anterior cingulate cortex) increased in response to cues that were semantically related to the prospective memory target, relative to unrelated cues (Hashimoto, Umeda, \& Kojima, 2011). A strength of the present approach is that it provides a robust measure of the timeline over which participants fixate on environmental cues, retrieve the intention, and subsequently begin to monitor for the upcoming target event. We found that even subtle cues in the form of semantically related images were effective in initiating strategic monitoring processes that were functionally related to prospective memory performance. Specifically, when participants in the prospective memory condition fixated on images during the ongoing task that were related to the prospective memory target, they were more likely to immediately engage an overt strategic monitoring process relative to when they fixated on unrelated images. This finding revealed a dynamic interaction between contextual cueing and the engagement of strategic monitoring processes (Scullin et al., 2013).

New paradigms, such as the one we have designed, can inform theoretical discussions in the prospective memory literature. For example, disagreement remains regarding the cognitive processes that are necessary for supporting prospective memory retrieval (Einstein \& McDaniel, 2010; Loft \& Remington, 2013; Smith, 2010). A recent claim that has sparked controversy is that individuals may not engage preparatory attention to monitor for upcoming prospective memory targets, even in tasks that are widely believed to require strategic monitoring to support the retrieval of future intentions (Heathcote, Loft, \& Remington, 2015; Loft \& Remington, 2013). This theoretical stance assumes that observed ongoing task costs in the typical laboratory task really reflect increased response thresholds associated with the prospective memory target, rather than participants engaging a preparatory monitoring process. By separating the ongoing task and the prospective memory target stimulus, the present paradigm partially addresses the potential response conflict 
confound noted by Loft and Remington in the typical measure of prospective memory monitoring cost. It is important to note, however, that the parameters of the task used in the present study do not allow for the calculation of an ongoing task cost, so it is unclear which strategic monitoring index provides the optimal measure of monitoring. Our goal was simply to provide an alternative measure of strategic monitoring that would focus on overt monitoring in situations in which the ongoing task and future intention are separate tasks.

Future research will be needed to determine how the task developed for the present study compares to the traditional event-based prospective memory task. Notably, the prospective memory target items are embedded within the ongoing task in the typical event-based prospective memory paradigm, whereas our task uses separate stimuli for the ongoing and prospective memory tasks. We consider this feature to be a key strength of our approach, because it enabled us to use eyetracking measures to isolate the strategic monitoring process, and in particular, the cue-driven monitoring process. Admittedly, it is unclear how our task would fit within the cue focality continuum related to intention retrieval in eventbased prospective memory tasks (Einstein et al., 2005). A task is considered to be focal if the ongoing task demands direct attention to the presence of the prospective memory target. In our approach, the separation of the ongoing and prospective memory tasks suggests that the prospective memory task is nonfocal to the ongoing task; however, embedding cues in the ongoing task that are semantically related to the prospective memory target did direct attention to the prospective memory task demand. Thus, our task (as well as many real-world prospective memory tasks) may contain both focal and nonfocal features, particularly when considering the impact of contextual cues in the environment. Furthermore, the separation of the ongoing and prospective memory tasks may bear more resemblance to the typical time-based prospective memory task, in which attention has to be diverted from the ongoing task to monitor an upcoming target event. In both time- and event-based tasks, the individual has to maintain two task sets (i.e., responding to the ongoing task and maintaining the future intention); however, time- and event-based tasks do exhibit distinct features, such as larger age deficits being observed in time-based tasks (for a review, see Henry, MacLeod, Phillips, \& Crawford, 2004) and differential patterns of activation within the rostral prefrontal cortex (Okuda et al., 2007). More research will be needed to determine how tasks that do not fit perfectly within the confines of the typical focal/nonfocal and time-/event-based distinctions relate to existing theories. Despite these differences, we contend that tasks such as the one we developed for the present study can provide important insight into the cognitive processes supporting prospective memory while considering the complexity and ambiguity of features in real-world prospective memory situations.
In general, we believe that the field of prospective memory has benefitted, and will continue to benefit, from the use of eyetracking techniques. The emergence of eyetracking approaches to study prospective memory has also led to new and interesting questions, such as how interruptions impact nurses in intensive care units (Grundgeiger, Sanderson, MacDougall, \& Venkatesh, 2010) and how visual gaze differs in prospective memory tasks, relative to visual search and free-viewing tasks (Hartwig, Schnitzspahn, Kliegel, Velichkovsky, \& Helmert, 2013). To our knowledge, the first eyetracking paradigm used in prospective memory research was developed by West, Carlson, and Cohen (2007), who used an eyetracker to assess fixation patterns as participants performed a visual search task in which prospective memory targets were embedded within the ongoing-task area. There, participants were unable to anticipate the location of prospective memory targets with relation to other ongoing-task-relevant stimuli. In contrast, our implementation of a distinctly separate area of the visual field for the prospective memory task allowed us to directly observe overt strategic monitoring processes. By annexing the prospective memory target to a separate area of the visual field, we were able to parse out visual searches for the prospective memory target from ongoing-task activity. Thus, the task used in the present study allows for a greater degree of confidence that fixations over the prospective memory area do not represent incidental visual wandering.

\section{Conclusions}

Traditional behavioral approaches for assessing prospective memory performance have led to great strides in our understanding of the cognitive mechanisms supporting prospective memory; however, the measurement of strategic monitoring used in these approaches has been indirect, leading to some ambiguity regarding what processes are being measured. The introduction of psychophysiological approaches, such as those measuring visual attention, offers the chance to directly observe the presence of overt strategic monitoring, or visually searching for the target. In the present study, the occurrence of each incidence of strategic monitoring was precisely recorded as participants engaged in a continuous visual search task. Moreover, our approach allowed us to observe the impact of contextual cues on the initiation of strategic monitoring. These findings are important for understanding how context interacts with the cognitive mechanisms supporting prospective memory retrieval. The new paradigm developed in the present study can be used to further inform current theoretical debates on the frequency and necessity of strategic monitoring 
processes, and how they facilitate the successful recollection of prospective memory intentions. ${ }^{2}$

Author note J.T.S. is now at the Department of Psychology, University of Tennessee at Chattanooga. E.A.C. is now at the Department of Psychological Sciences, Purdue University. We thank Kristina Byrd, Nikola Claud, Sara Stallings, Courtney Bramblett, and Lacey Gregory for their assistance with data collection and scoring. Portions of these data were presented at the conference of the American Psychological Association held in Orlando, Florida (August 2012), and the conference of the Southeastern Psychological Association held in Atlanta, Georgia (March 2013).

\section{References}

Brown, N. L., Hicks, J. L., \& Beck, M. R. (2014). The impact of visual attention on prospective memory. Paper presented at the 4th International Conference on Prospective Memory, Naples, Italy.

Dismukes, R. (2008). Prospective memory in aviation and everyday settings. In M. Kliegel, M. A. McDaniel, \& G. O. Einstein (Eds.), Prospective memory: Cognitive, neuroscience, developmental, and applied perspective (pp. 411-431). Mahwah: Erlbaum.

Einstein, G. O., \& McDaniel, M. A. (1996). Retrieval processes in prospective memory: Theoretical approaches and some new empirical findings. In M. Brandimonte, G. O. Einstein, \& M. A. McDaniel (Eds.), Prospective memory: Theory and applications (pp. 115141). Mahwah: Erlbaum.

Einstein, G. O., \& McDaniel, M. A. (2010). Prospective memory and what costs do not reveal about retrieval processes: A commentary on Smith, Hunt, McVay, and McConnell (2007). Journal of Experimental Psychology: Learning, Memory, and Cognition, 36, 1082-1088. doi:10.1037/a0019184

Einstein, G. O., McDaniel, M. A., Thomas, R., Mayfield, S., Shank, H., Morisette, N., \& Breneiser, J. (2005). Multiple processes in prospective memory retrieval: Factors determining monitoring versus spontaneous retrieval. Journal of Experimental Psychology: General, 134, 327-342. doi:10.1037/0096-3445.134.3.327

Foster, E. R., McDaniel, M. A., Repovš, G., \& Hershey, T. (2009). Prospective memory in Parkinson disease across laboratory and self-reported everyday performance. Neuropsychology, 23, 347358. doi: $10.1037 / \mathrm{a} 0014692$

Gold, J. M., Carpenter, C., Randolph, C., Goldberg, T. E., \& Weinberger, D. R. (1997). Auditory working memory and Wisconsin Card Sorting Test performance in schizophrenia. Archives of General Psychiatry, 54, 159-165. doi:10.1001/archpsyc.1997. 01830140071013

Grundgeiger, T., Sanderson, P., MacDougall, H. G., \& Venkatesh, B. (2010). Interruption management in the intensive care unit: Predicting resumption times and assessing distributed support. Journal of Experimental Psychology: Applied, 16, 317-334. doi: 10.1037/a0021912

Guajardo, N., \& Best, D. L. (2000). Do preschoolers remember what to do? Incentive and external cues in prospective memory. Cognitive Development, 15, 75-97. doi:10.1016/S0885-2014(00)00016-2

Harris, J. E., \& Wilkins, A. J. (1982). Remembering to do things: A theoretical framework and an illustrative experiment. Human Learning, 1, 123-136.

\footnotetext{
${ }^{2}$ The reported study is a replication of the original experiment, with a few minor modifications in the stimuli. Both experiments led to the same pattern of results and magnitude of effects, so we have only included the results of one experiment, for the sake of brevity.
}

Harrison, T. L., \& Einstein, G. O. (2010). Prospective memory: Are preparatory attentional processes necessary for a single focal cue? Memory \& Cognition, 38, 860-861. doi:10.3758/MC.38.7.860

Hartwig, J., Schnitzspahn, K. M., Kliegel, M., Velichkovsky, B. M., \& Helmert, J. R. (2013). I see you remembering: What eye movements can reveal about process characteristics of prospective memory. International Journal of Psychophysiology, 88, 193-199. doi:10. 1016/j.iipsych.2013.03.020

Hashimoto, T., Umeda, S., \& Kojima, S. (2011). Neural substrates of implicit cueing effect on prospective memory. NeuroImage, 54, 645-652.

Heathcote, A., Loft, S., \& Remington, R. W. (2015). Slow down and remember to remember! A delay theory of prospective memory costs. Psychological Review, 122, 376-410. doi:10.1037/a0038952

Henry, J. D., MacLeod, M. S., Phillips, L. H., \& Crawford, J. R. (2004). A meta-analytic review of prospective memory and aging. Psychology and Aging, 19, 27-39.

Henry, J. D., Rendell, P. G., Phillips, L. H., Dunlop, L., \& Kliegel, M. (2012). Prospective memory reminders: A laboratory investigation of initiation source and age effects. Quarterly Journal of Experimental Psychology, 65, 1274-1287. doi:10.1080/17470218. 2011.651091

Hicks, J. L., Marsh, R. L., \& Cook, G. I. (2005). An observation on the role of context variability in free recall. Journal of Experimental Psychology: Learning, Memory, and Cognition, 31, 1160-1164. doi:10.1037/0278-7393.31.5.1160

Horn, S. S., Bayen, U. J., \& Smith, R. E. (2011). What can the diffusion model tell us about prospective memory? Canadian Journal of Experimental Psychology, 65, 69-75. doi:10.1037/a0022808

Kim, P. Y., \& Mayhorn, C. B. (2008). Exploring students' prospective memory inside and outside the lab. American Journal of Psychology, 121, 241-254. doi:10.2307/20445459

Kuhlmann, B. G., \& Rummel, J. (2014). Context-specific prospectivememory processing: Evidence for flexible attention allocation adjustments after intention encoding. Memory \& Cognition, 42, 943 949. doi:10.3758/s13421-014-0405-2

Kvavilashvili, L., \& Fisher, L. (2007). Is time-based prospective remembering mediated by self-initiated rehearsals? Role of incidental cues, ongoing activity, age, and motivation. Journal of Experimental Psychology: General, 136, 112-132. doi:10.1037/0096-3445.136. 1.112

Loft, S., \& Remington, R. W. (2010). Prospective memory and task interference in a continuous monitoring dynamic display task. Journal of Experimental Psychology: Applied, 16, 145-157. doi: 10.1037/a0018900

Loft, S., \& Remington, R. W. (2013). Wait a second: Brief delays in responding reduce focality effects in event-based prospective memory. Quarterly Journal of Experimental Psychology, 66, 1432-1447.

Loft, S., Humphreys, M. S., \& Whitney, S. J. (2008). Control of access to memory: The use of task interference as a behavioral probe. Journal of Memory and Language, 58, 465-479. doi:10.1016/j.jml.2007.04. 002

Loft, S., Smith, R. E., \& Bhaskara, A. (2011). Prospective memory in an air traffic control simulation: External aids that signal when to act. Journal of Experimental Psychology: Applied, 17, 60-70. doi:10. 1037/a0022845

Marsh, R. L., Hicks, J. L., Cook, G. I., Hansen, J. S., \& Pallos, A. L. (2003). Interference to ongoing activities covaries with the characteristics of an event-based intention. Journal of Experimental Psychology: Learning, Memory, and Cognition, 29, 861-870. doi: 10.1037/0278-7393.29.5.861

McDaniel, M. A., \& Einstein, G. O. (2000). Strategic and automatic processes in prospective memory retrieval: A multiprocess framework. Applied Cognitive Psychology, 14, S127-S144. doi:10.1002/ acp. 775 
McDaniel, M. A., LaMontagne, P., Beck, S. M., Scullin, M. K., \& Braver, T. S. (2013). Dissociable neural routes to successful prospective memory. Psychological Science, 24, 1791-1800.

McNerney, M. W., \& West, R. (2007). An imperfect relationship between prospective memory and the prospective interference effect. Memory \& Cognition, 35, 275-282. doi:10.3758/BF03193448

Meier, B., \& Rey-Mermet, A. (2012). Beyond monitoring: After-effects of responding to prospective memory targets. Consciousness and Cognition, 21, 1644-1653. doi:10.1016/j.concog.2012.09.003

Nelson, D. L., McEvoy, C. L., \& Schreiber, T. A. (1998). The University of South Florida word association, rhyme, and word fragment norms [Database]. Retrieved from http://w3.usf.edu/ FreeAssociation/

Okuda, J., Fujii, T., Ohtake, H., Tsukiura, T., Yamadori, A., Frith, C. D., \& Burgess, P. W. (2007). Differential involvement of regions of rostral prefrontal cortex (Brodmann area 10) in time- and eventbased prospective memory. International Journal of Psychophysiology, 64, 233-246. doi:10.1016/j.ijpsycho.2006.09. 009

Park, D. C., Hertzog, C., Kidder, D. P., Morrell, R. W., \& Mayhorn, C. B. (1997). Effect of age on event-based and time-based prospective memory. Psychology and Aging, 12, 314-327. doi:10.1037/08827974.12.2.314

Rummel, J., \& Meiser, T. (2013). The role of metacognition in prospective memory: Anticipated task demands influence attention allocation strategies. Consciousness and Cognition, 22, 931-943. doi:10. 1016/j.concog.2013.06.006
Scullin, M. K., McDaniel, M. A., \& Einstein, G. O. (2010). Control of cost in prospective memory: Evidence for spontaneous retrieval processes. Journal of Experimental Psychology: Learning, Memory, and Cognition, 36, 190-203. doi:10.1037/a0017732

Scullin, M. K., McDaniel, M. A., \& Shelton, J. T. (2013). The Dynamic Multiprocess Framework: Evidence from prospective memory with contextual variability. Cognitive Psychology, 67, 55-71. doi:10. 1016/j.cogpsych.2013.07.001

Smith, R. E. (2003). The cost of remembering to remember in eventbased prospective memory: Investigating the capacity demands of delayed intention performance. Journal of Experimental Psychology: Learning, Memory, and Cognition, 29, 347-361. doi: 10.1037/0278-7393.29.3.347

Smith, R. E. (2010). What costs do reveal and moving beyond the cost debate: Reply to Einstein and McDaniel. Journal of Experimental Psychology: Learning, Memory, and Cognition, 36, 1089-1095. doi:10.1037/a0019183

Villa, K. (1998). Age and prospective memory: A neuropsychological account based on executive functioning. Dissertation Abstracts International, 58, 5146.

Wang, L., Altgassen, M., Liu, W., Xiong, W., Akgün, C., \& Kliegel, M. (2011). Prospective memory acrossadolescence: The effects of age and cue focality. Developmental psychology, 47(1), 226.

West, R., Carlson, L., \& Cohen, A. (2007). Eye movements and prospective memory: What the eyes can tell us about prospective memory. International Journal of Psychophysiology, 64, 269-277. doi:10. 1016/j.iipyscho.2006.09.006 